\title{
Understanding the rapidity dependence of the elliptic flow and the HBT radii at RHIC
}

\author{
M. Csanád*, T. Csörgố ${ }^{*}$, B. Lörstad ${ }^{* *}$ and A. Ster ${ }^{\dagger}$ \\ * Department of Atomic Physics, ELTE, Budapest, Pázmány P. 1/A, H-1117 \\ ${ }^{\dagger}$ MTA KFKI RMKI, H - 1525 Budapest 114, P.O.Box 49, Hungary \\ ${ }^{* *}$ Department of Physics, University of Lund, S-22362 Lund, Sweden
}

\begin{abstract}
The pseudo-rapidity dependence of the elliptic flow at various excitation energies measured by the PHOBOS Collaboration in Au+Au collisions at RHIC is one of the surprising results that has not been explained before in terms of hydrodynamical models. Here we show that these data are in agreement with theoretical predictions and satisfy the universal scaling relation predicted by the Buda-Lund hydrodynamical model, based on exact solutions of perfect fluid hydrodynamics. We also show a theoretical prediction on the rapidity and transverse momentum scaling of the HBT radii measured in heavy ion collisions, based on the Buda-Lund model.
\end{abstract}

\section{INTRODUCTION}

One of the unexpected results from experiments at the Relativistic Heavy Ion Collider (RHIC) is the relatively strong second harmonic moment of the transverse momentum distribution, referred to as the elliptic flow. Measurements of the elliptic flow by the PHENIX, PHOBOS and STAR collaborations (see refs. [1, 2, 3, 4]) reveal rich details in terms of its dependence on particle type, transverse and longitudinal momentum variables, on the centrality and the bombarding energy of the collision. In the soft transverse momentum region, these measurements at mid-rapidity are reasonably well described by hydrodynamical models $[5,6]$. However, the dependence of the elliptic flow on the longitudinal momentum variable pseudo-rapidity and its excitation function has resisted descriptions in terms of hydrodynamical models (but see their new description by the SPHERIO model [7]).

Here we show that these data are consistent with the theoretical and analytic predictions that are based on eqs. (1-6) of ref. [8], that is, on perfect fluid hydrodynamics.

We furthermore calculate rapidity dependent HBT (Bose-Einstein) radii in the framework of the model and make prediction on the universal scaling of these observables.

Our tool in describing the pseudorapidity-dependent elliptic flow and HBT radii is the Buda-Lund hydrodynamical model. The Buda-Lund hydro model [9] is successful in describing the BRAHMS, PHENIX, PHOBOS and STAR data on identified single particle spectra and the transverse mass dependent Bose-Einstein or HBT radii as well as the pseudorapidity distribution of charged particles in $\mathrm{Au}+\mathrm{Au}$ collisions both at $\sqrt{s_{\mathrm{NN}}}=130 \mathrm{GeV}$ [10] and at $\sqrt{s_{\mathrm{NN}}}=200 \mathrm{GeV}$ [11]. However the elliptic flow would be zero in an axially symmetric case, so we developed the ellipsoidal generalization of the model that describes an expanding ellipsoid with principal axes $X, Y$ and $Z$. Their derivatives with respect to proper-time (expansion rates) are denoted by $\dot{X}, \dot{Y}$ and $\dot{Z}$. 
The generalization goes back to the original one, if the transverse directed principal axes of the ellipsoid are equal, ie $X=Y$ (and also $\dot{X}=\dot{Y}$ ).

The deviation from axial symmetry can be measured by the momentum-space eccentricity,

$$
\varepsilon_{p}=\frac{\dot{X}^{2}-\dot{Y}^{2}}{\dot{X}^{2}+\dot{Y}^{2}}
$$

The exact analytic solutions of hydrodynamics (see ref. $[8,12,13]$ ), which form the basis of the Buda-Lund hydro model, develop Hubble-flow for late times, ie $X \rightarrow_{\tau \rightarrow \infty} \dot{X} \tau$, so the momentum-space eccentricity $\varepsilon_{p}$ nearly equals space-time eccentricity $\varepsilon$.

Let us introduce $\Delta \eta$ additionally. It represents the elongation of the source expressed in units of space-time rapidity. Let us consider furthermore that at the freeze-out $\tau \Delta \eta=$ $Z$ and $Z \approx \dot{Z} \tau$, and so $\Delta \eta \approx \dot{Z}$

Hence, in this paper we extract space-time eccentricity $(\varepsilon)$, average transverse flow $\left(u_{t}\right)$ and longitudinal elongation $(\Delta \eta)$ from the data, instead of $\dot{X}, \dot{Y}$ and $\dot{Z}$.

In the time dependent hydrodynamical solutions, these values evolve in time, however, it was show in ref. [14] that $\dot{X}, \dot{Y}$ and $\dot{Z}$, and so $\varepsilon, u_{t}$ and $\Delta \eta$ become constants of the motion in the late stages of the expansion.

\section{RAPIDITY DEPENDENT ELLIPTIC FLOW}

The result for the elliptic flow (under certain conditions detailed in ref [15]) is the following simple universal scaling law:

$$
v_{2}=\frac{I_{1}(w)}{I_{0}(w)}
$$

The model predicts an universal scaling: every $v_{2}$ measurement is predicted to fall on the same universal scaling curve $I_{1} / I_{0}$ when plotted against $w$.

This means, that $v_{2}$ depends on any physical parameter (transverse or longitudinal momentum, center of mass energy, centrality, type of the colliding nucleus etc.) only through the (universal) scaling paremeter $w$.

Here $w$ is the scaling variable, defined by

$$
w=\frac{p_{t}^{2}}{4 \bar{m}_{t}}\left(\frac{1}{T_{*, y}}-\frac{1}{T_{*, x}}\right),
$$

and

$$
\begin{aligned}
T_{*, x} & =T_{0}+\bar{m}_{t} \dot{X}^{2} \frac{T_{0}}{T_{0}+\bar{m}_{t} a^{2}}, \\
T_{*, y} & =T_{0}+\bar{m}_{t} \dot{Y}^{2} \frac{T_{0}}{T_{0}+\bar{m}_{t} a^{2}},
\end{aligned}
$$

and

$$
\bar{m}_{t}=m_{t} \cosh \left(\eta_{s}-y\right)
$$


Here $a=\langle\Delta T / T\rangle_{t}$ measures the temperature gradient in the transverse direction, at the freeze-out, $m_{t}$ is the transverse mass, $T_{0}$ the central temperature at the freeze-out, while $\eta_{s}$ is the space-time rapidity of the saddle-point (point of maximal emittivity). This saddlepoint depends on the rapidity, the longitudinal expansion, the transverse mass and on the central freeze-out temperature:

$$
\eta_{s}-y=\frac{y}{1+\Delta \eta \frac{m_{t}}{T_{0}}}
$$

where $y=0.5 \log \left(\frac{E+p_{z}}{E-p_{z}}\right)$ is the rapidity.

More details about the ellipsoidally symmetric model and its result on $v_{2}(\eta)$ can be found in ref. [15].

Eq. 2 depends, for a given centrality class, on rapidity $y$ and transverse mass $m_{t}$. Before comparing our result to the $v_{2}(\eta)$ data of PHOBOS, we thus performed a saddle point integration in the transverse momentum variable and performed a change of variables to the pseudo-rapidity $\eta=0.5 \log \left(\frac{|p|+p_{z}}{|p|-p_{z}}\right)$, similarly to ref. [16]. This way, we have evaluated the single-particle invariant spectra in terms of the variables $\eta$ and $\phi$, and calculated $v_{2}(\eta)$ from this distribution, a procedure corresponding to the PHOBOS measurement decribed in ref. [1].

We have found that the essential fit parameters are $\varepsilon$ and $\Delta \eta$, and the quality of the fit is insensitive to the precise value of $T_{0}, a$ and $u_{t}$. These parameters dominate the azimuthalaveraged single particle spectra as well as the HBT (Bose-Einstein) radii, however they only marginally influence $v_{2}$. Their precise value is irrelevant in a broad region of values and does not influence the confidence level of the $v_{2}(\eta)$ fits. Hence we have fixed their values as given in the caption of table 1 . We also excluded points with large rapidity from the fits in case of lower center of mass energies.

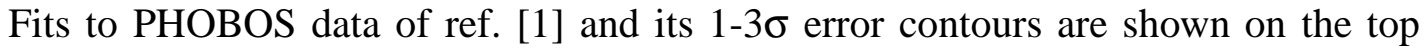
two panels of fig. 1. The fitting package is available at ref. [17]. Bottom panel of fig. 1 demonstrates that the investigated PHOBOS data points follow the theoretically predicted scaling law.

Table 1. Results of fits to PHOBOS data of ref. [1]. Both space-time eccentricity $(\varepsilon)$ and longitudinal elongation $(\Delta \eta)$ increase with increasing $\sqrt{s_{\mathrm{NN}}}$. Remaining parameters were fixed as follows: $T_{0}=175 \mathrm{MeV}, a=1.19$ and $u_{t}=1.64$.

\begin{tabular}{|l|c|c|c|c|}
\hline & $19.6 \mathrm{GeV}$ & $62.4 \mathrm{GeV}$ & $130 \mathrm{GeV}$ & $200 \mathrm{GeV}$ \\
\hline$\varepsilon$ & $0.294 \pm 0.029$ & $0.349 \pm 0.008$ & $0.376 \pm 0.005$ & $0.394 \pm 0.006$ \\
$\Delta \eta$ & $1.70 \pm 0.25$ & $2.16 \pm 0.05$ & $2.46 \pm 0.04$ & $2.56 \pm 0.04$ \\
$\chi^{2} / \mathrm{NDF}$ & $1.84 / 11$ & $20.1 / 13$ & $34.8 / 15$ & $27.5 / 15$ \\
conf. level & $100 \%$ & $21.4 \%$ & $1.00 \%$ & $7.03 \%$ \\
\hline
\end{tabular}



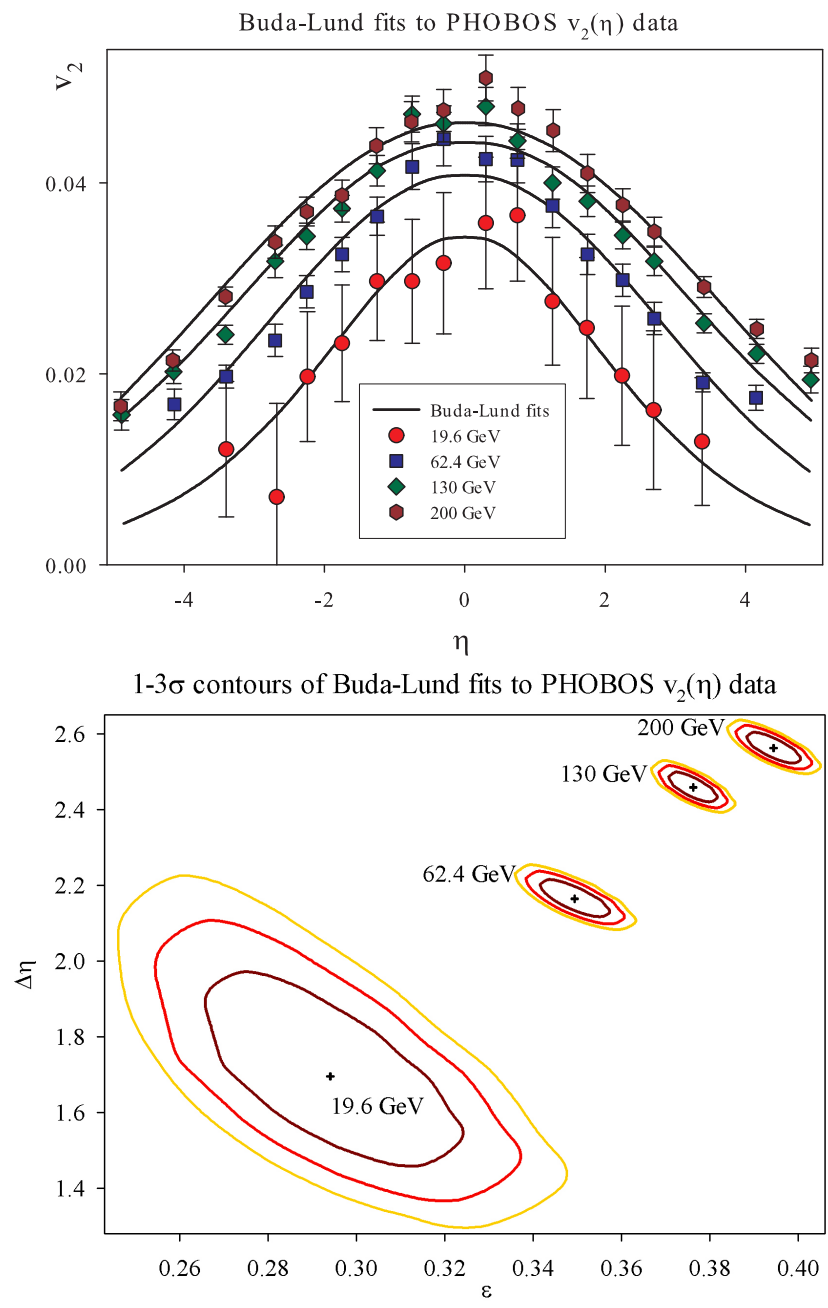

PHOBOS $\mathrm{v}_{2}$ versus scaling variable $\mathrm{w}$

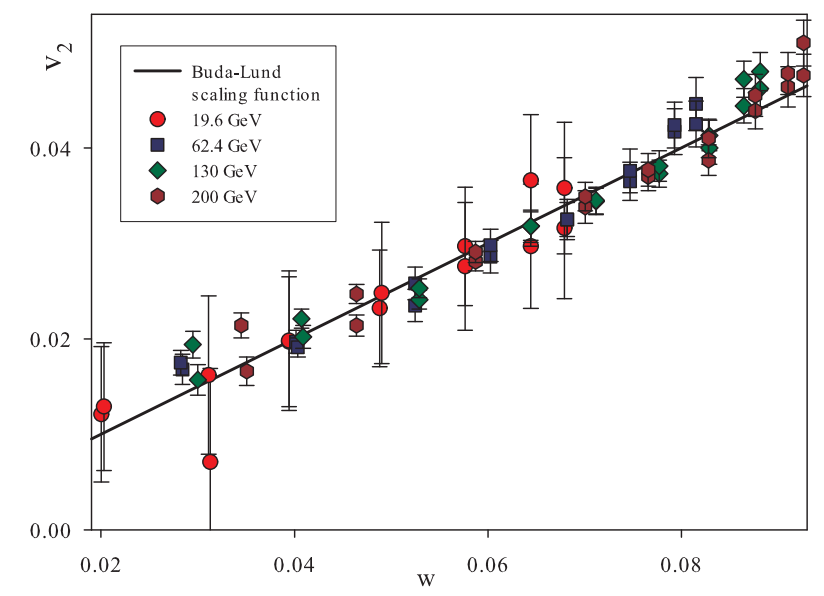

Figure 1. Top: PHOBOS data on the pseudorapidity dependence of the elliptic flow [1], at various center of mass energies, with Buda-Lund fits. Middle: Error contours of the fits. Bottom: Elliptic flow versus scaling variable $w$ is plotted. The data points of ref. [1] show theoretically predicted [15]) universal scaling, when plotted against the universal scaling variable $w$. 

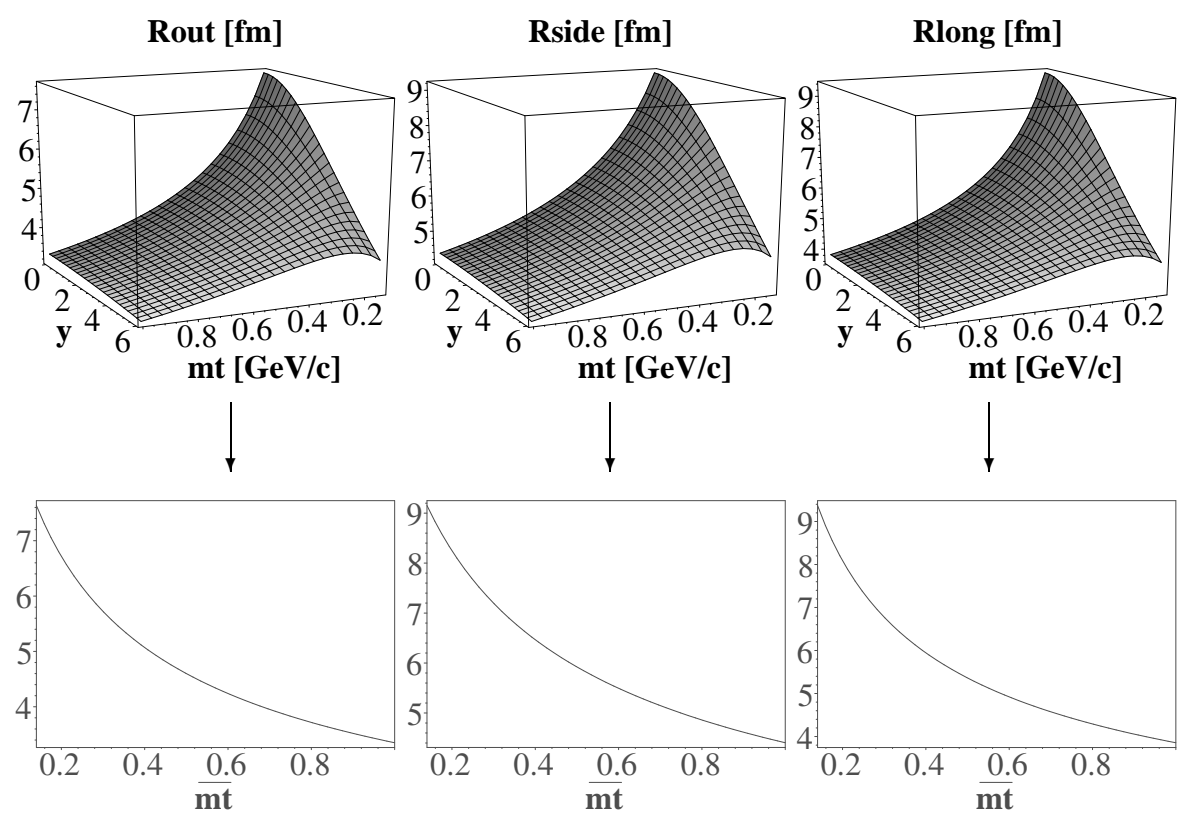

Figure 2. Upper panel: $R_{\text {out }}, R_{\text {side }}$ and $R_{\text {long }}$ as a function of rapidity $y$ and transverse mass $m_{t}$. Lower panel: The two-dimensional $R\left(m_{t}, y\right)$ functions are predicted to show a scaling behavior, insofar as they depend only on scaling variable $\overline{m_{t}}$.

\section{RAPIDITY DEPENDENT HBT RADII}

In the framework of the model we can also calculate the HBT radii. In the simplest case, where system of ellipsoidal expansion equals the out-side-longitudinal coordinate system:

$$
\begin{aligned}
& R_{\text {out }}^{2}=X^{2}\left(1+\frac{\bar{m}_{t}\left(a^{2}+\dot{X}^{2}\right)}{T_{0}}\right)^{-1}, \\
& R_{\text {side }}^{2}=Y^{2}\left(1+\frac{\bar{m}_{t}\left(a^{2}+\dot{Y}^{2}\right)}{T_{0}}\right)^{-1}, \\
& R_{\text {long }}^{2}=Z^{2}\left(1+\frac{\bar{m}_{t}\left(a^{2}+\dot{Z}^{2}\right)}{T_{0}}\right)^{-1} .
\end{aligned}
$$

This means, that the HBT radii depend on transverse mass and rapidity only through the scaling paremeter $\overline{m_{t}}$, as illustrated on fig. 2 . This behavior could easily be checked by measurement of rapidity and transverse momentum dependence of the HBT radii and comparing this data to the present prediction of the Buda-Lund model. Such a comparision could be a further test of perfect fluid hydrodynamics. 


\section{CONCLUSIONS}

In summary, we have shown that the excitation function of the pseudorapidity dependence of the elliptic flow in $\mathrm{Au}+\mathrm{Au}$ collisions is well described with the formulas that are predicted by the Buda-Lund type of hydrodynamical calculations.

We have provided a quantitative evidence of the validity of the perfect fluid picture of soft particle production in $\mathrm{Au}+\mathrm{Au}$ collisions at $\mathrm{RHIC}$ but also show here that this perfect fluid extends far away from mid-rapidity.

We also suggest a further test of perfect fluid hydrodynamics at large rapidities, expressed by eqs. 8-10 and illustrated by fig. 2 .

The universal scaling of PHOBOS $v_{2}(\eta)$, expressed by eq. 2 and illustrated by fig. 1 provides a successful quantitative as well as qualitative test for the appearence of a perfect fluid in $\mathrm{Au}+\mathrm{Au}$ collisions at various colliding energies at RHIC.

\section{ACKNOWLEDGMENTS}

It is our pleasure to acknowledge the inspiring discussions with Roy Lacey, Stephen Manly and Gábor Veres. This research was supported by the NATO Collaborative Linkage Grant PST.CLG.980086, by the Hungarian - US MTA OTKA NSF grant INT0089462 and by the OTKA grant T038406 .

\section{REFERENCES}

1. B. B. Back et al. [PHOBOS Collaboration], Phys. Rev. Lett. 94, 122303 (2005)

2. S. S. Adler et al. [PHENIX Collaboration], Phys. Rev. Lett. 91, 182301 (2003)

3. C. Adler et al. [STAR Collaboration], Phys. Rev. Lett. 87, 182301 (2001)

4. P. Sorensen [STAR Collaboration], J. Phys. G 30, S217 (2004)

5. K. Adcox et al. [PHENIX Collaboration], Nucl. Phys. A 757, 184 (2005)

6. J. Adams et al. [STAR Collaboration], Nucl. Phys. A 757, 102 (2005)

7. F. Grassi, Y. Hama, O. Socolowski and T. Kodama, J. Phys. G 31, S1041 (2005).

8. T. Csörgő, L. P. Csernai, Y. Hama and T. Kodama, Heavy Ion Phys. A 21, 73 (2004)

9. T. Csörgő and B. Lörstad, Phys. Rev. C 54 (1996) 1390

10. M. Csanád, T. Csörgő, B. Lörstad, A. Ster, Act. Phys. Pol. B35, 191 (2004)

11. M. Csanád, T. Csörgő, B. Lörstad and A. Ster, Nukleonika 49, S45 (2004)

12. S. V. Akkelin, T. Csörgő, B. Lukács, Y. M. Sinyukov, M. Weiner, PLB 505, 64 (2001)

13. T. Csörgő, hep-ph/0111139.

14. T. Csörgő and J. Zimányi, Heavy Ion Phys. 17, 281 (2003)

15. M. Csanád, T. Csörgő and B. Lörstad, Nucl. Phys. A 742, 80 (2004)

16. D. Kharzeev and E. Levin, Phys. Lett. B 523, 79 (2001) [arXiv:nucl-th/0108006].

17. http://www.phenix.bnl.gov/viewcvs/offline/analysis/budalund/ 